\title{
Subcellular localisation of lipoproteins of Vibrio vulnificus by the identification of outer membrane vesicles components
}

\author{
Yan-Jiao Zhang $\cdot$ Huiyuan Lin $\cdot$ Pan Wang $\cdot$ Chang Chen $\cdot$ Shiyong Chen $(\mathbb{D}$
}

Received: 2 January 2018/Accepted: 26 April 2018/Published online: 2 May 2018

(C) Springer International Publishing AG, part of Springer Nature 2018

\begin{abstract}
Vibrio vulnificus, a Gram-negative halophilic bacterium, is an opportunistic human pathogen that is responsible for the majority of seafood-associated deaths worldwide. Lipoproteins are important components of the bacterial cell envelope and have been shown to be involved in a wide variety of cellular processes. Little is known about the localisation or transport mechanism of lipoproteins in $V$. vulnificus. To assess the localisation of lipoproteins in $V$. vulnificus, we tested two established techniques for
\end{abstract}

Yan-Jiao Zhang and Huiyuan Lin equally contributed to this work.

Electronic supplementary material The online version of this article (https://doi.org/10.1007/s10482-018-1092-y) contains supplementary material, which is available to authorized users.

Y.-J. Zhang · H. Lin · P. Wang · S. Chen $(\bowtie)$

Shandong Province Key Laboratory of Applied

Mycology, School of Life Sciences, Qingdao Agricultural

University, Shandong 266109, China

e-mail: shiyongchen@hotmail.com

\section{S. Chen}

Shandong Engineering Research Center for Aquatic

Animal Immune Preparation, Marine Science and

Engineering College, Qingdao Agricultural University,

Shandong 266109, China

C. Chen $\cdot$ S. Chen

Key Laboratory of Tropical Marine Bio-resources and Ecology, Chinese Academy of Sciences,

Guangzhou 510301, China the rapid separation of membrane-associated proteins: detergent extraction with Sarkosyl and outer membrane vesicles (OMVs) preparation. The results showed that Sarkosyl extraction was not useful for the separation of lipoproteins from the different membranes of $V$. vulnificus. On the other hand, we confirmed that OMVs produced by $V$. vulnificus contained lipoproteins from the outer but not the inner membrane. Analysis of the OMVs components confirmed the localisation of several well-known lipoproteins to membranes that were different from expected, based on their predicted functions. Using this technique, we found that Asp at position +2 of mature lipoproteins can function as an inner membrane retention signal in $V$. vulnificus. Interestingly, the Escherichia coli " +2 rule" does not apply to the $V$. vulnificus lipoprotein IlpA (G2D) mutant, as a Ser to Asp mutation at position +2 of IlpA did not affect its outer membrane localisation. Furthermore, an IlpA tether-mRFP chimeric lipoprotein and its G2D mutant also behaved like IlpA. Together, these results suggest that the sorting rule of lipoproteins in $V$. vulnificus might be different from that in E. coli.

Keywords +2 rule Lipoprotein - Localisation Sorting · Outer membrane vesicles · Vibrio vulnificus 


\section{Introduction}

Vibrio vulnificus, a Gram-negative halophilic bacterium, is ubiquitous in estuaries and coastal environments and can cause human illness, known as vibriosis (Baker-Austin and Oliver 2017).This serious human pathogen is responsible for the majority of seafoodassociated deaths worldwide (Jones and Oliver 2009) and for vibriosis in different species of aquatic animals (Amaro et al. 2015). Treatment of infected humans and aquatic animals is becoming increasingly challenging as $V$. vulnificus has begun to develop antibiotic resistance (Heng et al. 2017). Many aspects of its biology, genomics, virulence and epidemiology still need to be fully elucidated.

Bacterial lipoproteins are a subset of membrane proteins with their membrane association mediated by a lipid moiety attached to the N-terminal Cysteine residue of the mature protein (Braun and $\mathrm{Wu} 1994$; Nakayama et al. 2012). Lipoproteins are typically essential components of the Gram-negative cell envelope and important virulence factors (KovacsSimon et al. 2011). Most lipoproteins in Escherichia coli and other Enterobacteriaceae are found on the periplasmic side of the inner or outer membranes. They are transferred from the inner membrane (IM) to the outer membrane (OM) by the Lol (localization of lipoproteins) machinery (Okuda and Tokuda 2011). Localisation is determined by the amino acid immediately following the lipidated Cys, in the +2 position (Yamaguchi et al. 1988). The " +2 rule" for E. coli lipoprotein sorting states that Asp at the +2 position acts as an IM retention signal, while any other amino acid in that position results in OM localisation. Results from a variety of recent studies now suggest that the surface exposure of lipoproteins in Gram-negative bacteria is a widespread phenomenon (Konovalova and Silhavy 2015; Wilson and Bernstein 2016). Unfortunately, few of the surface lipoprotein secretion mechanisms have been studied in detail.

$V$. vulnificus is predicted to encode $\sim 100$ lipoproteins, based on algorithms that search for cyscontaining 'lipobox' motifs in genome sequences and other criteria (Babu et al. 2006; http://www.mrclmb.cam.ac.uk/genomes/dolop/compgen.shtml). Interestingly, a reported surface lipoprotein, immunogenic lipoprotein A (IlpA), has dual functions as an adhesin and a major immunostimulant in $V$. vulnificus-host cell interactions (Goo et al. 2007; Lee et al. 2010). However, until recently, little work had been carried out on the other lipoproteins in $V$. vulnificus. The Lol pathway components LolA-E and the lipoprotein maturation enzymes (Lgt, Lsp, and Lnt) (Sutcliffe et al. 2012) are found in V. vulnificus genome. The detailed mechanism of lipoprotein biogenesis, including lipoprotein maturation and transport to the $\mathrm{OM}$, remains elusive. Developing a technique to assess localisation of lipoproteins is an important step for the study of the lipoprotein transport mechanism in $V$. vulnificus and potentially for vaccine development against this bacterial infection.

There are two commonly used techniques for subcellular fractionation of membrane-associated proteins: detergent extraction with Sarkocyl and outer membrane vesicles (OMVs) preparation. Sarkosyl extraction is widely used to separate the OM and IM systems and localise membrane-associated proteins in Gram-negative bacteria. Membrane proteins can be obtained by selective solubilisation of IM components from crude membrane fractions by detergent treatment. This technique was applied in Vibrio cholerae to determine lipoprotein localisations (Bose and Taylor 2005; Morris et al. 2008). OMVs are small, spherical bilayered vesicles released into the extracellular environment from the OM of Gram-negative bacteria (Beveridge 1999). OMVs can perform a variety of functions including acquisition of nutrients, elimination of competing organisms, cell-to-cell signaling, aid bacterial survival, and modulation of host immune response, and are considered a novel secretion system (Lee et al. 2008; Jan 2017). Recent proteomic analyses of Gram-negative bacterial OMVs indicate that, compared with cellular proteins, OMVs are enriched with proteins associated with the extracellular region, outer membrane, and periplasm, and are depleted for inner membrane proteins (Lee et al. 2016). The localisation of membrane associated proteins, including lipoproteins, in Borrelia burgdorferi (Skare et al. 1995; Schulze and Zückert 2006) and E.coli (Moriel et al. 2010) has been achieved by analysing the components of whole cells and OMV fractions.

In this study, we aimed to develop a technique to assess the localisation of lipoproteins in V. vulnificus. We achieved this by testing two techniques commonly used for the separation of membrane associated proteins, Sarkosyl extraction and OMVs preparation. We then used the OMVs technique to determine if the " +2 rule" sorting rule from $E$. coli can be applied to $V$. 
vulnificus. Our results show that the sorting rule of lipoproteins in $V$. vulnificus might be different from that of E. coli.

\section{Materials and methods}

Bacterial strains and growth conditions

The strains and plasmids used in this study are listed in Table 1. V. vulnificus ATCC 27562 was cultured in Luria-Bertani (LB) broth or on LB agar at $30{ }^{\circ} \mathrm{C}$. To prepare OMVs, V. vulnificus was cultured overnight in LB broth as a starter culture. Overnight cell cultures were inoculated $(1: 100, \mathrm{v} / \mathrm{v})$ into fresh LB medium and allowed to grow to late exponential phase $\left(\mathrm{OD}_{600}=1.0\right)$. When appropriate, antibiotics were added at the following concentrations: chlorampheni$\operatorname{col}(17 \mu \mathrm{g} / \mathrm{ml})$, and polymyxin E $(12.5 \mu \mathrm{g} / \mathrm{ml})$. E. coli DH5 $\alpha$ and SM10 $\lambda$ pir were cultured as described above except ampicillin $(100 \mu \mathrm{g} / \mathrm{ml})$ was added when needed.

\section{Construction of plasmids}

All plasmids used in this study are derivatives of pSCT32 (Zhang et al. 2017) and were generated using ClonExpress One Step Cloning Kit (Vazyme Biotech). The expression of the recombinant proteins was driven by an IPTG-inducible promoter. All proteins were modified with a C-terminal $\mathrm{His}_{6}$-tag to facilitate subsequent immunoblot analysis and purification. Both vector and insert DNA fragments were amplified using Phusion (Thermo Scientific) high-fidelity DNA

Table 1 Bacterial strains and plasmids used in this study

\begin{tabular}{|c|c|c|}
\hline Strain or plasmid & Description & Source or references \\
\hline \multicolumn{3}{|l|}{ Strains } \\
\hline \multicolumn{3}{|l|}{ V. vulnificus } \\
\hline ATCC 27562 & Type strain & Lab collection \\
\hline \multicolumn{3}{|l|}{ E. coli } \\
\hline $\mathrm{DH} 5 \alpha$ & supE44lacU169(lacZM15)hsdR17 recA lendA1 gyrA96 thi-1 relA1 & Lab collection \\
\hline SM10 & & Simon et al. (1983) \\
\hline Plasmids & thi thr leu tonA lacY supE recA[RP4-2-Tc::Mu] $\lambda$ pirR6K Km ${ }^{\mathrm{r}}$ & \\
\hline pSCT32 & A broad host range regulatable expression vector & Yan-Jiao et al. (2017) \\
\hline pSCT24 & ilpA in pSCT32 & This study \\
\hline pSCT25 & ompU in $\mathrm{pSCT} 32$ & This study \\
\hline pSCT33 & $w z a$ in $\mathrm{pSCT} 32$ & This study \\
\hline pSCT34 & $w z a-(T 2 D)$ in $\mathrm{pSCT} 32$ & This study \\
\hline pSCT35 & ilpA-(G2D) in pSCT32 & This study \\
\hline pSCT36 & $m f p$ in $\mathrm{pSCT} 32$ & This study \\
\hline pSCT40 & lolB in $\mathrm{pSCT} 32$ & This study \\
\hline pSCT41 & lolE in pSCT32 & This study \\
\hline pSCT43 & lsp in pSCT32 & This study \\
\hline pSCT46 & lptE in $\mathrm{pSCT} 32$ & This study \\
\hline pSCT47 & lptE-(G2D) in pSCT32 & This study \\
\hline pSCT48 & bamD in $\mathrm{pSCT} 32$ & This study \\
\hline pSCT49 & ilpA32-mrfp fusion in pSCT32 & This study \\
\hline pSCT50 & ilpA32-mrfp-(G2D) fusion in pSCT32 & This study \\
\hline pSCT109 & AOT11_RS11575 in pSCT32 & This study \\
\hline
\end{tabular}


polymerase to perform recombination cloning. The vector fragment was amplified from pSCT32 with the pSCTNdeI-rev and Linker His-fwd primers. Protein genes (ompU, ilpA, wza, lsp, bamD, lolB, lptE, lolE, $m f p$ and AOT11_RS11575) were amplified from $V$. vulnificus ATCC 27562 genomic DNA with genespecific primers (Table 2). The V. vulnificus IlpA signal peptide was inserted to replace the original signal peptide of the LolB protein for improved expression. After ligation, the products were transfected to $E$. coli competent cells. The bacterial colonies were collected and confirmed by DNA sequencing.

The IlpA32-mrfp fusion constructs, in which monomeric red fluorescence protein ( $m r f p$ ) was fused to the truncated lipoprotein IlpA tether peptide IlpA32, were generated by sequence overlap extension PCR (SOEPCR). Fragments containing the IlpA tether peptide IlpA32, amplified from $V$. vulnificus ATCC 27562 genomic DNA with IlpA-fwd and IlpA32-rev, and $m r f p$ gene, amplified from pRJS1077 (Schulze et al. 2010) with IlpA32-mRFP-fwd and mRFP-rev, were fused using IlpA-fwd and mRFP-rev. The amplicons were then ligated with the pSCT32 vector fragment and transformed into $E$. coli.

The +2 site mutation was introduced into lipoproteins Wza, LptE, IlpA and IlpA32-mRFP with primer pairs Wza T2D-fwd and Wza T2D-rev, LptE G2Dfwd and LptE G2D-rev, IlpA G2D-fwd and IlpA G2Drev, using the QuikChange-II XL site-directed mutagenesis kit (Stratagene), resulting in plasmids pSCT34, pSCT47, pSCT35 and pSCT50 respectively.

All plasmids were verified by PCR and DNA sequencing (Sangon Biotech., Shanghai, China).The resulting plasmids were transformed into $E$. coli SM10 $\lambda$ pir and transferred into V. vulnificus ATCC 27562 by conjugation. The exconjugants were selected on LB agar containing chloramphenicol and polymyxin E.

Preparation of antiserum of OmpU and IlpA

The recombinant form of the OmpU (rOmpU) protein was overexpressed in E. coli BL21(DE3) carrying pSCT 25 by adding IPTG at a concentration of $0.1 \mathrm{mM}$ and purified using the HisPur Cobalt affinity column as directed by the manufacturer (Thermo Scientific).The recombinant form of IlpA (rIlpA) protein was overexpressed in E. coli BL21 (DE3) carrying plasmid pSCT24 and purified in the same manner as the rOmpU protein. Purified rOmpU or rllpA (each $120 \mu \mathrm{g}$ ) was used to immunise Kunming mice (4week-old, female) for the production of polyclonal antisera. The antisera against OmpU or IlpA were obtained from the immunised mice 1 week after the third immunisation and used for immunoblot analysis.

\section{Cellular fractionation}

V. vulnificus ATCC 27562 cells were obtained by centrifuging the bacterial cultures at $10,000 \times g$ for $15 \mathrm{~min}$ at $4{ }^{\circ} \mathrm{C}$. Cells were washed with $10 \mathrm{mM}$ Tris$\mathrm{Cl}$ ( $\mathrm{pH}$ 8.0). Washed cells were resuspended and broken by sonication. Unbroken cells and debris were removed by centrifugation $\left(10,000 \times g, 15 \mathrm{~min}, 4{ }^{\circ} \mathrm{C}\right)$. Fractionation of whole-cell lysates into total membrane (TM), outer membrane (OM), and inner membrane (IM) fractions was accomplished by the method of Bose and Taylor (2005). Protein estimation was carried out using the BCA protein assay kit (Pierce) with bovine serum albumin as a standard.

\section{Purification of $V$. vulnificus OMVs}

OMVs were prepared from exponential phase cultures of strain ATCC 27562 as previously described with minor modifications (Wai et al. 2003). Briefly, culture supernatants were obtained by centrifuging the bacterial cultures at $10,000 \times g$ for $15 \mathrm{~min}$ at $4{ }^{\circ} \mathrm{C}$. The supernatant was collected and filtered through $0.22 \mu \mathrm{m}$ pore-size membrane filters (Millipore) to remove remaining bacterial cells. The supernatants were then centrifuged at $150,000 \times g$ for $4{ }^{\circ} \mathrm{C}$ in a $45 \mathrm{Ti}$ rotor (Beckman Instruments, Inc.).The resulting pellets, which contained the OMVs, were resuspended in PBS (pH 7.6). Protein estimation was carried out using the BCA protein assay kit (Pierce).

\section{Transmission electron microscopy}

The purified OMVs were adsorbed onto carbon-coated nickel 400-mesh grids. The grids were allowed to dry, stained with $2 \%$ phosphotungstic acid $(\mathrm{pH} 7.0)$ for 5 min, and washed with PBS. The morphology of the OMVs was examined under a Hitachi TEM System $\mathrm{HC}-1$ at $80 \mathrm{kV}$. 
Table 2 Oligonucleotides used in this study

\begin{tabular}{|c|c|c|}
\hline Name & Sequence $\left(5^{\prime}-3^{\prime}\right)$ & Description \\
\hline $\begin{array}{l}\text { pSCT NdeI- } \\
\text { rev }\end{array}$ & CATATGGTCTGTTTCCTGTGTG & Vector cloning forward primer \\
\hline $\begin{array}{l}\text { LinkerHis- } \\
\text { fwd }\end{array}$ & CCCGGGGGTTCAGGAGCTCATCATCATCACCATCACTAAG & Vector cloning reverse primer \\
\hline OmpU-fwd & CACACAGGAAACAGACCATATGAAAAAGACATTAATCGCACTTTC & $\begin{array}{l}\text { ompU gene cloning forward } \\
\text { primer }\end{array}$ \\
\hline OmpU-rev & GCTCCTGAACCCCCGGGGAAGTCGTAACGTAGACCTAG & ompU gene cloning reverse primer \\
\hline IlpA-fwd & CACACAGGAAACAGACCATATGAAATTTAGCCTCAAAGG & ilpA gene cloning forward primer \\
\hline IlpA-rev & GCTCCTGAACCCCCGGGCCAGCCTTTTACTACGCC & ilpA gene cloning reverse primer \\
\hline Wza-fwd & CACACAGGAAACAGACCATATGCTTTCAAAAGCGTTAGAAAG & $w z a$ gene cloning forward primer \\
\hline Wza-rev & GCTCCTGAACCCCCGGGCGGCCACGTTTTAACGCGTAG & $w z a$ gene cloning reverse primer \\
\hline Lsp-fwd & CACACAGGAAACAGACCATATGAGCGGTAAAGCGCAG & $l s p$ gene cloning forward primer \\
\hline Lsp-rev & GCTCCTGAACCCCCGGGCTTCTCTTCTTCCTTTTTCCG & $l s p$ gene cloning reverse primer \\
\hline BamD-fwd & CACACAGGAAACAGACCATATGAGCGGTAAAGCGCAG & $\begin{array}{l}\text { bamD gene cloning forward } \\
\text { primer }\end{array}$ \\
\hline BamD-rev & GCTCCTGAACCCCCGGGCTTCTCTTCTTCCTTTTTCCG & bamD gene cloning reverse primer \\
\hline LolB-fwd & CACACAGGAAACAGACCATATGCTCTTGCCGCTGTTTATG & lolB gene cloning forward primer \\
\hline LolB-rev & GCTCCTGAACCCCCGGGTTTAGCGTCCACTTACTCAC & lolB gene cloning reverse primer \\
\hline LptE-fwd & CACACAGGAAACAGACCATATGCGTTTTACGTCCGTG & lptE gene cloning forward primer \\
\hline LptE-rev & GCTCCTGAACCCCCGGGCTGATTACTTGAGTTGACGG & lptE gene cloning reverse primer \\
\hline MFP-fwd & CACACAGGAAACAGACCATATGCGCAAACCCCAGATAAC & $m f p$ gene cloning forward primer \\
\hline MFP-rev & GCTCCTGAACCCCCGGGAATGCCCTCCTCGCGTAC & $m f p$ gene cloning reverse primer \\
\hline LolE-fwd & CACACAGGAAACAGACCATATGATTTCTTCCTTGGCG & lolE gene cloning forward primer \\
\hline LolE-rev & GCTCCTGAACCCCCGGGTTTACTGCTCAGCACGGC & lolE gene cloning reverse primer \\
\hline IlpA32-rev & TTTGCTCGTGTCAACGGC & $\begin{array}{l}\text { IlpA tether truncation (to aa } 32 \text { ) } \\
\text { reverse primer }\end{array}$ \\
\hline $\begin{array}{l}\text { IlpA32- } \\
\text { mRFP-fwd }\end{array}$ & GCCGTTGACACGAGCAAAGACGTCATCAAGGAGTTCATG & $\begin{array}{l}\text { IlpA tether-mRFP fusion cloning } \\
\text { forward primer }\end{array}$ \\
\hline mRFP-rev & GCTCCTGAACCCCCGGGGGCGCCGGTGGAGTGGCG & $m r f p$ gene cloning reverse primer \\
\hline $\begin{array}{l}\text { Wza T2D- } \\
\text { fwd }\end{array}$ & GCCGGTTGTGATGTACCCGGCTCACATCTATCC & $\begin{array}{l}\text { Wza tether T2D forward } \\
\text { mutagenic primer }\end{array}$ \\
\hline $\begin{array}{l}\text { Wza T2D- } \\
\text { rev }\end{array}$ & CCGGGTACATCACAACCGGCAAGCAAGGC & $\begin{array}{l}\text { Wza tether T2D reverse } \\
\text { mutagenic primer }\end{array}$ \\
\hline $\begin{array}{l}\text { LptE G2D- } \\
\text { fwd }\end{array}$ & GTCTGCATGTGATTTTCATTTACGTGGTGAGTACTC & $\begin{array}{l}\text { LptE tether T2D forward } \\
\text { mutagenic primer }\end{array}$ \\
\hline $\begin{array}{l}\text { LptE G2D- } \\
\text { rev }\end{array}$ & ATGAAAATCACATGCAGACAACATGGC & $\begin{array}{l}\text { LptE tether T2D reverse } \\
\text { mutagenic primer }\end{array}$ \\
\hline $\begin{array}{l}\text { IlpA G2D- } \\
\text { fwd }\end{array}$ & AGCAGGTTGTGACGAAAAAGCCGTTGACACG & $\begin{array}{l}\text { IlpA tether T2D forward } \\
\text { mutagenic primer }\end{array}$ \\
\hline $\begin{array}{l}\text { IlpA G2D- } \\
\text { rev }\end{array}$ & GCTTTTTCGTCACAACCTGCTAATACCAATGTTG & $\begin{array}{l}\text { IlpA tether T2D reverse } \\
\text { mutagenic primer }\end{array}$ \\
\hline $\begin{array}{l}\text { RS11575- } \\
\text { fwd }\end{array}$ & CACACAGGAAACAGACCATATGTGTAACTTACTCAAAATCGTC & $\begin{array}{l}R S 11575 \text { gene cloning forward } \\
\text { primer }\end{array}$ \\
\hline $\begin{array}{l}\text { RS11575- } \\
\text { rev }\end{array}$ & GCTCCTGAACCCCCGGGACGCATCGTATCGACTTG & $\begin{array}{l}\text { RS11575 gene cloning reverse } \\
\text { primer }\end{array}$ \\
\hline
\end{tabular}


SDS-PAGE and immunoblot analysis

Proteins were separated by sodium dodecyl sulfatepolyacrylamide gel electrophoresis (SDS-PAGE) and visualised by Coomassie blue staining. For immunoblots, proteins were electrophoretically transferred to a PVDF membrane (Millipore) using a Transblot semidry transfer cell (Bio-Rad). Membranes were blocked and incubated with antibodies in 5\% dry milk, $20 \mathrm{mM}$ Tris-500 mM NaCl, $0.05 \%$ Tween-20. The antibodies used were mouse polyclonal antisera against $\mathrm{OmpU}$ (dilution, 1:20,000) and IlpA (dilution, 1:10,000). The secondary antibody was goat-anti-mouse $\operatorname{IgG}(\mathrm{H}+\mathrm{L})$ (Sigma). Proteins overexpressed in V. vulnificus such as Wza, BamD, Lsp, LptE, MFP, LolB, and LolE, which were tagged with a hexahistidine epitope tag, were detected directly with a nickel-activated HisProbe-HRP conjugate (Pierce). SuperSignal West Dura substrate (Pierce) was used for the chemiluminescent detection of HRP-labeled proteins.

\section{Results}

Sarkosyl extraction cannot be used for localisation of lipoproteins in $V$. vulnificus

Sarkosyl has been shown to be useful for dividing crude envelope samples from $V$. cholerae into distinct inner and outer membrane protein profiles (Lohia et al. 1984).More recently, this technique has been used to localise lipoproteins in V. cholerae (Bose and Taylor 2005; Morris et al. 2008). Therefore, we first attempted to investigate the subcellular localisation of lipoproteins in V. vulnificus using a Sarkosyl-based membrane fractionation protocol (Bose and Taylor, 2005).

V. vulnificus strain ATCC 27562, which expressed a C-terminal His $_{6}$-tag Wza protein, was grown to $\mathrm{OD}_{600}$ 2.0. The bacteria were harvested and subcellular fractionation was performed as described in materials and methods. The TM, OM and IM fractions were isolated from the strain and run on an SDSPAGE gel. To verify the correct lipoprotein localisation, one known $\mathrm{OM}$ protein, $\mathrm{OmpU}$, and two known OM lipoproteins, Wza and IlpA, were identified by immunoblotting analysis. The $\mathrm{His}_{6}$-tagged Wza was identified using an anti-Histag reagent HisProbe, while OmpU and IlpA were identified using specific
WC TM OM IM

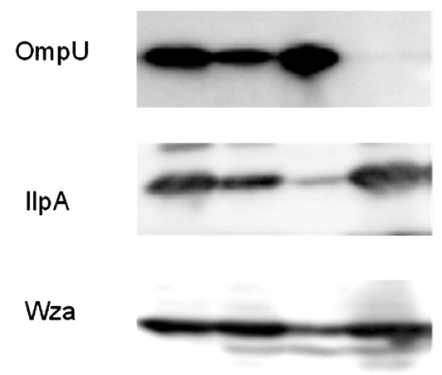

Fig. 1 Localization of proteins by Sarcacyl extraction. $V$. vulnificus cells harbaring pSCT33 were grown in LB medium to $\mathrm{OD}_{600}=1$ as described in materials and methods. The bacteria were harvested and subcellular fractionation was performed. Total membrane (TM), outer membrane (OM) and inner membrane (IM) were isolated from the V. vulnificus cell and immunoblotting analysis of whole cell (WC), TM, OM and IM samples were performed as described in the Materials and methods section. OmpU an outer membrane protein, IlpA an OM lipoprotein, Wza an OM lipoprotein

antisera (Fig. 1). All three proteins, Wza, OmpU and IlpA, were clearly detected in the whole cell samples and the TM sample, indicating that these three proteins are membrane-associated proteins. A lack of OmpU in the IM fraction and an enrichment of OmpU in the OM fraction indicated that these samples are enriched specifically for OM proteins. However, as determined by Western blotting (Fig. 1), the two known OM lipoproteins, IlpA and Wza, were detected predominantly in the IM, rather than the OM fraction. These results were not consistent with previously published protein functional and localisation studies (Goo et al. 2007; Wright et al. 2001), which indicated that Sarkosyl-based membrane fractionation method is not suitable for separation and localisation of lipoproteins in $V$. vulnificus.

\section{V. vulnificus produces OMVs with OM lipoprotein}

Lipoproteins in B. burgdorferi (Skare et al. 1995; Dowdell et al. 2017) and E. coli (Moriel et al. 2010) have been localised by analysis of the components of OMVs. However, proteomic analyses of Gram-negative bacterial OMVs indicate that bacterial OMVs still carried IM components (Lee et al. 2016). Therefore, we investigated whether OMVs of $V$. vulnificus can be used to localise lipoproteins.

First we isolated OMVs from exponential phase cultures of V. vulnificus strain ATCC 27562. Vesicle 
a

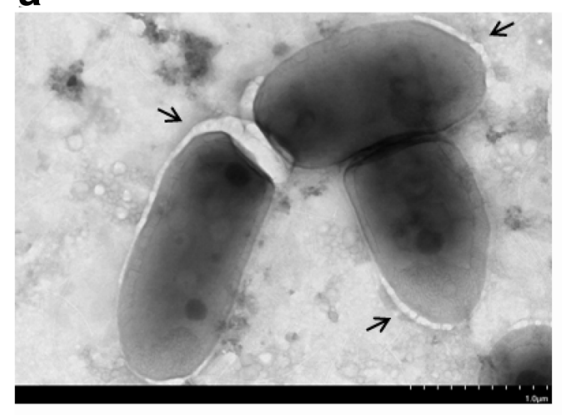

C

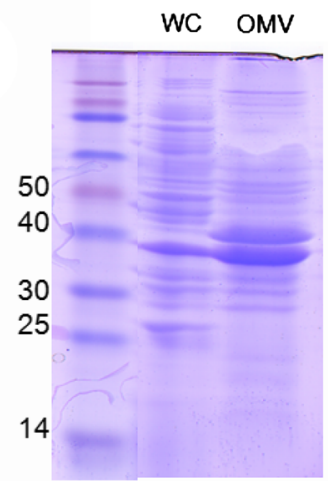

Fig. 2 V. vulnificus produces OMVs carrying OM lipoprotein. a Transmission electron micrograph (TEM) of negatively stained V. vulnificus strain ATCC 27562 cultured in logarithmic phase of growth shows the presence of vesicles (indicated by arrows). Bar $=1 \mu \mathrm{m}$. b TEM of negatively stained OMVs purified from culture broth of $V$. vulnificus strain ATCC 27562. Two types of OMVs are seen in the photographs. Most vesicles have a single membrane, but occasionally vesicles with two membranes are observed (black arrow). Both vesicle chain-like

production by $V$. vulnificus was confirmed by transmission electron microscopy (Fig. 2a, b).We observed free vesicles and vesicle chain-like morphotypes with diameters ranging from 30 to $250 \mathrm{~nm}$. Most vesicles had a single membrane, but occasionally vesicles with two membranes were observed. Subsequently, purified OMVs were subjected to SDS-PAGE and immunoblotting. OmpU and IlpA were used again as OM markers for detection of OMVs. The known IM protein Lsp, the dedicated lipoprotein signal peptidase, was used as a control to assess the purity of the OMV preparation, as it should be absent from the OMV preparation. The immunoreactive bands of OmpU and IlpA were observed in both whole cell and OMVs samples (Fig. 2d). There was no IM marker protein Lsp found in the OMV samples, indicating that the presence of OmpU and IlpA in the b
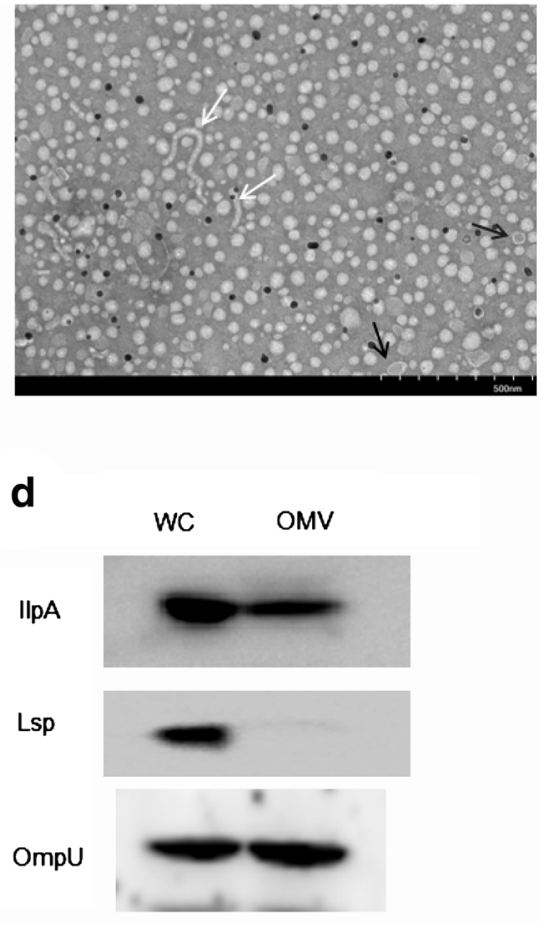

morphotype (indicated by white arrows) and free vesicles were observed in the purified vesicle fraction. Bar $=500 \mathrm{~nm}$. c $V$. vulnificus whole-cell lysates (WC) and purified OMVs were separated by SDS-PAGE and the gel was stained with Coomassie blue. d Immunoblotting analysis of protein contents in WC and OMVs fractions from $V$. vulnificus. The OMVs contain bacterial proteins localized to the outer membrane. IlpA an OM lipoprotein, $L s p$ an IM signal peptidase, $O m p U$ an OM protein

OMVs was not a consequence of contamination with IM proteins during the secretion of $V$. vulnificus OMVs. Thus, immunoblot analyses confirmed that the purified OMVs were derived from the OM of $V$. vulnificus and carried OM lipoproteins.

Localisation of lipoproteins by isolation of OMVs in $V$. vulnificus

In order to further confirm the association of the $\mathrm{OM}$ lipoproteins with OMVs, we expanded our study to include five additional proteins, whose functions and localisations are conserved across Gram-negative bacteria. It was reported that OMVs released by normally growing $E$. coli contain a low concentration of lipoproteins (Wensink and Witholt 1981). To improve the sensitivity of protein detection, we 
decided to overexpress lipoproteins in $V$. vulnificus. Three known $V$. vulnificus OM lipoproteins, BamD (Onufryk et al. 2005; Malinverni et al. 2006), LolB (Tanaka et al. 2001) and LptE (Wu et al. 2006), a known IM protein LolE (the Lol pathway ABC transporter component; Yakushi et al. 2000) and two predicted IM lipoproteins, membrane fusion protein (MFP) and AOT11_RS11575, were overexpressed with a C-terminal $\mathrm{His}_{6}$-tag in strain ATCC 27562.

OMVs were isolated from exponential $(6 \mathrm{~h})$ phase cultures of $V$. vulnificus cells harbouring different protein overexpression plasmids. V. vulnificus wholecell lysates and purified OMVs were separated by SDS-PAGE, then immunoblotting was used to screen for the $\mathrm{His}_{6}$-tagged proteins. The three known OM lipoproteins, BamD (Fig. 3a), LolB (Fig. 3b) and LptE (Fig. 3c), were detected in the OMVs samples. While the predicted IM lipoprotein MFP (Fig. 3e), AOT11_RS11575 (Fig. 3f) and the IM marker protein
LolE (Fig. 3d) were almost undetectable in the OMVs samples. Taken together, these results demonstrated that OM lipoproteins but not IM lipoproteins are present in the $V$. vulnificus OMVs preparations.

The "+2 rule" does not apply to $V$. vulnificus lipoprotein IlpA (G2D) mutant

In E. coli, lipoprotein localisation follows the so called " +2 rule", where an N-terminal Asp +2 is predictive of IM localisation, while any other residue at that position stipulates OM localisation (Yamaguchi et al. 1988). Of the nearly 100 predicted lipoproteins from the $V$. vulnificus YJ016 genome, only two have an $\mathrm{Asp}+2$ at the N-terminus (Babu et al. 2006). Because we expect a greater proportion of lipoproteins to be localised to the IM, we wondered if the E. coli " +2 rule" was applicable to $V$. vulnificus. To assess if the $\mathrm{N}$-terminal Asp+2 plays a crucial role in localisation
Fig. 3 Localisation of lipoproteins and Lol gene by isolation of OMVs in $V$. vulnificus. $V$. vulnificus cells harbaring different protein over-expression plasmids were grown in LB medium. OMVs were isolated from exponential phase cultures as described in materials and methods. V. vulnificus whole-cell lysates and purified OMVs were separated by SDS-PAGE. The expression of Histagged lipoprotein BamD (a), LolB (b), LptE (c), Lol pathway $\mathrm{ABC}$ transporter component LolE (d), predicted IM lipoprotein MFP (e) and AOT11_RS11575 (f) were detected by Western blotting with Hisprobe. Samples were normalized to the $\mathrm{OM}$ protein $\mathrm{OmpU}$ a

WC

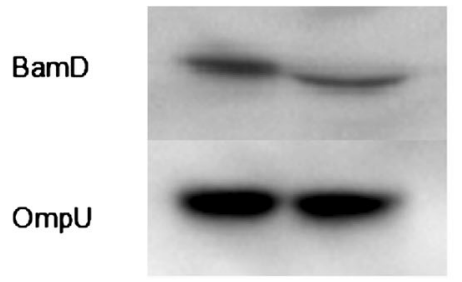

C

LptE

OmpU

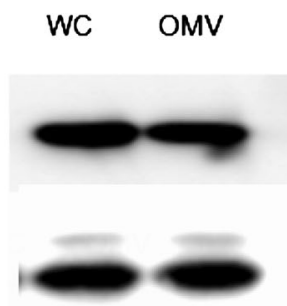

e

MFP

OmpU b

LolB

OmpU

d

LolE

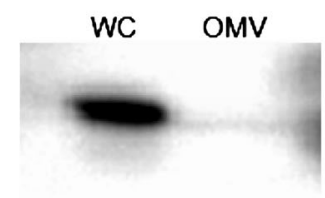

OmpU

f

AOT11_RS11575

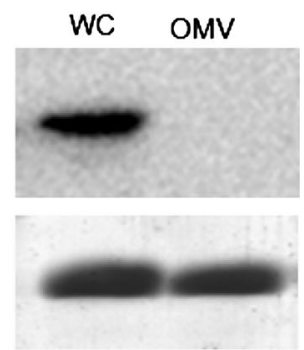


a

\begin{tabular}{|c|c|c|}
\hline IIpA : & $\begin{array}{l}+1+2 \\
\text { C-G-E-K-A-V-D-T-S-K- }\end{array}$ & IIPA \\
\hline IlpA (G2D) : & C-D-E-K-A-V-D-T-S-K-- & IIpA \\
\hline Wza : & C-T- V-P- G-S-H-L-S-T-- & LptE \\
\hline Wza (T2D) : & C-D-V-P-G-S-H-L-S-T-- & LptE \\
\hline LptE : & C-G-F-H- L-R-G-E-Y-S-- & Wza \\
\hline LptE (G2D) : & C-D-F-H- L-R-G-E-Y-S-- & Wza \\
\hline IlpA32-mRFP: & C-G-E-K-A-V-D-T-S-K-- & mRFP \\
\hline IlpA32-mRFP (G2D) : & C-D-E-K-A-V-D-T-S-K-- & MRFP \\
\hline
\end{tabular}

b

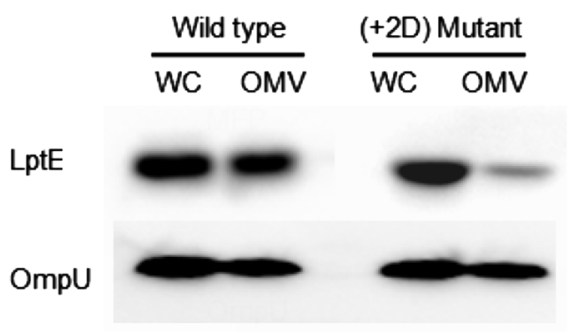

Fig. 4 " +2 rule" cannot apply to V. vulnificus IlpA (G2D) mutant. a A schematic overview of lipoproteins and their tether peptide " +2 " site mutant, as well as the fusion proteins created from IlpA and mRFP. Tether peptides are shown in one-letter amino acid codes. Amino acid positions relative to the acylmodified Cys residue (defined as +1 ) are shown above the wt peptide sequence. His $_{6}$-tagged lipoprotein and their “+2" site mutants, IlpA and IlpA (G2D) (b), LptE and LptE (G2D) (c), Wza and Wza (T2D) (d), IlpA32-mRFP and IlpA32-mRFP

of lipoproteins in $V$. vulnificus, the second residues in three mature OM lipoproteins, Wza, LptE, and IlpA were mutated to aspartates.

Wza, LptE, and IlpA tether peptide mutants (Fig. 4a) were generated by modifying existing Wza, LptE, and IlpA expression plasmids through oligomediated site-directed mutagenesis as described in the Materials and Methods. The resulting plasmids were used to transform V. vulnificus strain ATCC 27562. The localisation of +2 site mutants was identified by immunoblot analysis of the OMVs. While overexpressed wild type Wza and LptE were found in the
C

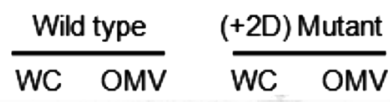

Wza

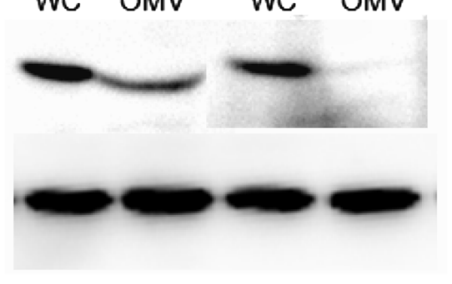

d

OmpU

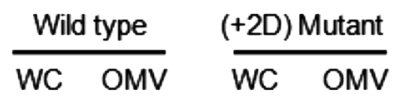

IIpA

OmpU

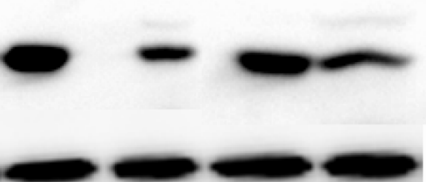

IlpA32-mRFP

OmpU

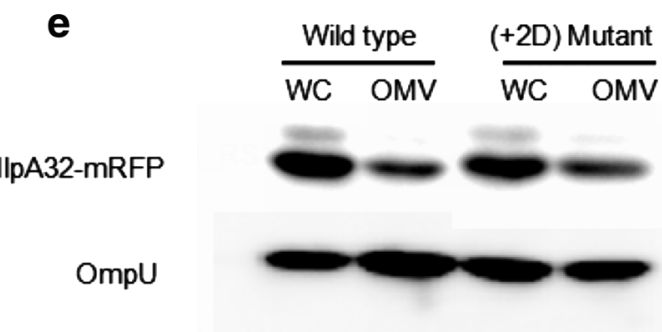

(G2D) (e), were expressed in V. vulnificus, respectively. $V$. vulnificus cells harbaring different protein over-expression plasmids were grown in LB medium. OMVs were isolated from exponential phase culturesas as described in materials and methods. V. vulnificus whole-cell lysates and purified OMVs were analyzed by SDS-PAGE and immunoblotting. His ${ }_{6}$-tagged lipoprotein was detected with Hisprobe. OmpU were detected with the antisera. Samples were normalized to the OM protein OmpU

OMVs preparations, the Wza (T2D) and LptE (G2D) mutants were mislocalised, being almost undetectable in the OMVs samples (Fig. 4b, c). This could indicate that Asp at position +2 functions as an IM retention signal in $V$. vulnificus. However, similar to the wild type IlpA protein, the IlpA (G2D) mutant was localised predominantly to the OM, suggesting the " +2 rule" from $E$. coli does not always hold true for $V$. vulnificus.

In order to confirm that the lipoprotein sorting signal is present in the $\mathrm{N}$-terminal tether residues of IlpA, we constructed an IlpA32-mRFP chimeric 
lipoprotein, comprising the signal peptide, $10 \mathrm{~N}$ terminal residues of the mature region of IlpA and a reporter protein, mRFP (Fig. 4a). The resulting fusion lipoprotein was well expressed and located in the outer membrane of V. vulnificus (Fig. 4e). The mRFP reporter fusion to the wild type $\mathrm{N}$-terminal lipopeptide behaved like the wild type IlpA lipoprotein, showing that the N-terminus of lipoprotein IlpA is sufficient for proper OM localisation, which is consistent with other reports in E.coli and B. burgdorferi (Ghrayeb and Inouye 1984; Schulze and Zückert 2006). The IlpA32mRFP fusion lipoprotein retained its predominantly OM location when +2 site Gly was mutated to Asp (Fig. 4d).This result confirmed the localisation pattern of the IlpA (G2D) mutant and showed that the C-terminal globular protein region does not affect the final destinations of lipoproteins.

Together, these experiments demonstrated that while Asp can serve as the canonical IM retention signal in $V$. vulnificus, the ' +2 rule' does not apply to all $V$. vulnificus lipoproteins.

\section{Discussion}

The established techniques developed for separating membrane-associated proteins in bacteria include sucrose density gradient centrifugation, detergent extraction of the crude envelope and OMVs preparation. The sucrose density gradient centrifugation technique has been commonly used in studies of Gram-negative bacteria. However, in some cases, a clean separation of the outer and inner membranes was not achieved by this technique. For example, incomplete separation of the two membranes by this technique were found in $V$. cholerae, which might due to a tighter linking of the inner and outer membranes than in other Gram-negative bacteria (Kelley and Parker 1981; Lohia et al. 1984).

Techniques based on selective solubilisation by detergents are widely used for separating OM and IM associated proteins, with the detergent-extracted outer membrane sample having lower phospholipid content (Lugtenberg and Van Alphen 1983; Lohia et al. 1984). In the current study, we could not detect the OM lipoprotein markers, IlpA and Wza, in Sarkosylextracted OM fractions of $V$. vulnificus. Detergent treatment of $\mathrm{OM}$ preparations might result in the loss of lipoproteins together with the phospholipids.
Therefore, Sarkosyl-based membrane fractionation is not suitable for separation and localisation of lipoproteins in $V$. vulnificus, although it worked in V. cholerae (Bose and Taylor 2005; Morris et al. 2008).

A wide variety of Gram-negative bacteria constitutively secrete OMVs during growth (Beveridge 1999; Jan 2017). OMVs are spherical, bilayered proteolipids and are composed of outer membrane proteins, lipopolysaccharide, outer membrane lipids, periplasmic proteins, cytoplasmic proteins, DNA, RNA and other factors associated with virulence (Lee et al. 2008).In some cases, OMVs also contain the components from the inner membrane and cytoplasm (Kulp and Kuehn 2010). The bacterial pathogens $V$. cholerae and $V$. vulnificus were reported to secrete OMVs (Chatterjee and Das 1967; Kim et al. 2010). Proteomic analysis of $V$. cholerae OMVs showed that of 90 identified proteins, $8 \%$ were IM associated and $6 \%$ were cytoplasmic proteins (Altindis et al. 2014). It was reported that OMVs released by normally growing $E$. coli contain very little lipoprotein (Wensink and Witholt 1981). Therefore, to verify our lipoprotein localisation method, we overexpressed and then identified the localisation of seven known $V$. vulnificus lipoproteins including two predicted IM lipoproteins, MFP and AOT11_RS11575. Our experimental data showed that $V$. vulnificus OMVs contained low amounts of IM proteins. Two overexpressed IM marker proteins, Lsp and LolE, were undetectable in the OMVs fractions. All lipoproteins included in this study were localised as expected based on their known functions; however, signal levels in OMVs fraction were weaker than those in whole cell samples after normalization with OmpU.

The E. coli-derived lipoprotein sorting rules seems to be conserved for bacteria in the family Enterobacteriaceae such as Salmonella enterica serovar Typhimurium, Shigella flexneri, Yersinia pseudotuberculosis, Erwinia carotovora, and Klebsiella oxitoca (Lewenza et al. 2006), but didn't fully extend to other $\gamma$-proteobacteria. Studies in Pseudomonas aeruginosa showed that an Asp at position +2 could function as an IM retention signal, but that Lys and Ser at position +3 and +4 , respectively, were the innate IM signals of lipoprotein MexA (Narita and Tokuda 2007). Our experimental data in V. vulnificus showed that two predicted IM proteins, MFP and AOT11_RS11575, carrying the Asp+2 residue in the $\mathrm{N}$-terminal tether, and two OM lipoprotein mutants, 
Wza (T2D) and LptE (G2D), all located to the IM, which indicated that Asp +2 can function as an IM retention signal in V. vulnificus. However, a Gly to Asp mutation at position +2 of the OM lipoprotein IlpA tether did not affect its localisation to the OM. It was also found that, using mRFP as a localisation reporter, an IlpA tether-mRFP chimeric lipoprotein and its G2D mutant behaved like IlpA and localised to the OM. In E. coli, the presence of the His or Lys residues at position +3 lowered the stringency of IM retention by an Asp+2 signal (Gennity and Inouye 1991). When the residue at position +3 was Asp, Glu or Gln, lipoproteins with Asp +2 were strongly retained in spheroplasts even in the presence of a saturating concentration of LolA (Terada et al. 2001). However, in the V. vulnificus system, the IlpA (G2D) mutant and the IlpA32-mRFP (G2D) could be transported to the $\mathrm{OM}$ with $\mathrm{Asp}+2$ and Glu+3 residues in their N-terminal tether. This led us to hypothesise that different residues in the tether sequence might govern the sorting of lipoproteins in $V$. vulnificus.

Interestingly, few putative IM lipoproteins with an $\mathrm{Asp}+2$ at the $\mathrm{N}$-terminus, could be found in the list of lipoproteins predicted by genome-wide computational analysis of the $V$. vulnificus genome (Babu et al. 2006). However, some IM-specific lipoproteins with a residue other than Asp at position +2 , such as the membrane fusion proteins (MFPs), are present in the V. vulnificus lipoproteome (Fig. S1). The membrane fusion proteins (MFPs) are N-terminally anchored to the IM by either a lipid moiety or a helical signal anchor sequence and are a major component of resistance-nodulation-division (RND) 2-type multidrug efflux pumps (Paulsen et al. 1996) and should therefore be localized to the IM. However, none of the MFP lipoproteins in Fig. S1 have Asp at the position +2 . These observations suggest that lipoprotein sorting signals differ between $V$. vulnificus and E. coli, a situation previously observed from the study of $P$. aeruginosa MexA (Narita and Tokuda 2007). The precise sorting rules of lipoproteins to the $\mathrm{OM}$ in $V$. vulnificus remain to be determined.

In conclusion, we have developed a convenient and precise technique to identify the membrane association of $V$. vulnificus lipoproteins, which might be applicable to other systems. Future studies will continue to define sorting rules for lipoproteins targeted to different membranes in $V$. vulnificus, and to test the involvement of the Lol machinery in the secretion of surface lipoproteins. While the establishment of this technique will allow us to localise additional $V$. vulnificus lipoproteins, the finding that this species has novel sorting rules encourages us to fully elucidate the precise molecular mechanism of lipoprotein transport in $V$. vulnificus.

Acknowledgements This work was supported by the National Natural Science Foundation of China (31372564, 31300005), Shandong Province Key Research and Development Program (Public welfare) (2017NC210001), Natural Science Foundation of Shandong Province (ZR2013CM028), and Open Project Program of LMB, CAS (2017-09). We thank Christina Azodi for critical reading and advice on the manuscript.

Conflict of interest The authors declare that they have no conflict of interest.

\section{References}

Altindis E, Fu Y, Mekalanos JJ (2014) Proteomic analysis of Vibrio cholerae outer membrane vesicles. Proc Natl Acad Sci USA 111:E1548-1556

Amaro C, Sanjuán E, Fouz B, Pajuelo D, Lee CT, Hor LI, Barrera R (2015) The fish pathogen Vibrio vulnificus biotype 2: epidemiology, phylogeny, and virulence factors involved in warm-water vibriosis. Microbiol Spectr 3(3):VE-0005-2014

Babu MM, Priya ML, Selvan AT, Madera M, Gough J, Aravind L, Sankaran K (2006) A database of bacterial lipoproteins (DOLOP) with functional assignments to predicted lipoproteins. J Bacteriol 188:2761-2773

Baker-Austin C, Oliver JD (2017) Vibrio vulnificus-new insights into a deadly opportunistic pathogen. Environ Microbiol. https://doi.org/10.1111/1462-2920.13955

Beveridge TJ (1999) Structures of gram-negative cell walls and their derived membrane vesicles. J Bacteriol 181:4725-4733

Bose N, Taylor RK (2005) Identification of a TcpC-TcpQ outer membrane complex involved in the biogenesis of the toxincoregulated pilus of Vibrio cholerae. J Bacteriol 187:2225-2232

Braun V, Wu HC (1994) Lipoproteins: structure function, biosynthesis and model for protein export. New Comp Biochem 27:319-341

Chatterjee SN, Das J (1967) Electron microscopic observations on the excretion of cell-wall material by Vibrio cholerae. J Gen Microbiol 49:1-11

Dowdell AS, Murphy MD, Azodi C, Swanson SK, Florens L, Chen S, Zückert WR (2017) Comprehensive spatial analysis of the Borrelia burgdorferi lipoproteome reveals a compartmentalization bias toward the bacterial surface. J Bacteriol 199:e0658-16

Gennity JM, Inouye M (1991) The protein sequence responsible for lipoprotein membrane localization in Escherichia coli 
exhibits remarkable specificity. J Biol Chem 266:16458-16464

Ghrayeb J, Inouye M (1984) Nine amino acid residues at the $\mathrm{NH} 2$-terminal of lipoprotein are sufficient for its modification, processing, and localization in the outer membrane of Escherichia coli. J Biol Chem 259:463-467

Goo SY, Han YS, Kim WH, Lee KH, Park SJ (2007) Vibrio vulnificus IlpA-induced cytokine production is mediated by Toll-like receptor 2. J Biol Chem 282:27647-27658

Heng SP, Letchumanan V, Deng CY, Ab Mutalib NS, Khan TM, Chuah LH, Chan KG, Goh BH, Pusparajah P, Lee LH (2017) Vibrio vulnificus: an environmental and clinical burden. Front Microbiol 8:997. https://doi.org/10.3389/ fmicb.2017.00997

Jan AT (2017) Outer membrane vesicles (OMVs) of gramnegative bacteria: a perspective update. Front Microbiol 8:1053. https://doi.org/10.3389/fmicb.2017.01053

Jones MK, Oliver JD (2009) Vibrio vulnificus: disease andpathogenesis. Infect Immun 77:1723-1733

Kelley JT, Parker CD (1981) Identification and preliminary characterisation of Vibrio cholerae outer membrane proteins. J Bacteriol 145:1018-1024

Kim YR, Kim BU, Kim SY, Kim CM, Na HS, Koh JT, Choy HE, Rhee JH, Lee SE (2010) Outer membrane vesicles of Vibrio vulnificus deliver cytolysin-hemolysin VvhA into epithelial cells to induce cytotoxicity. Biochem Biophys Res Commun 399:607-612

Konovalova A, Silhavy TJ (2015) Outer membrane lipoprotein biogenesis: Lol is not the end. Philos Trans R Soc Lond B 370(1679):20150030. https://doi.org/10.1098/rstb.2015. 0030

Kovacs-Simon A, Titball RW, Michell SL (2011) Lipoproteins of bacterial pathogens. Infect Immun 79:548-561

Kulp A, Kuehn MJ (2010) Biological functions and biogenesis of secreted bacterial outer membrane vesicles. Annu Rev Microbiol 64:163-184

Lee EY, Choi DS, Kim KP, Gho YS (2008) Proteomics in gramnegative bacterial outer membrane vesicles. Mass Spectrom Rev 27:535-555

Lee KJ, Lee NY, Han YS, Kim J, Lee KH, Park SJ (2010) Functional characterization of the IlpA protein of Vibrio vulnificus as an adhesin and its role in bacterial pathogenesis. Infect Immun 78:2408-2417

Lee J, Kim OY, Gho YS (2016) Proteomic profiling of Gramnegative bacterial outer membrane vesicles: current perspectives. Proteomics Clin Appl 10:897-909

Lewenza S, Pugsley AP, Vidal-Ingigliardi D (2006) Direct visualization of red fluorescent lipoproteins indicates conservation of the membrane sorting rules in the family Enterobacteriaceae. J Bacteriol 188:3516-3524

Lohia A, Chatterjee AN, Das J (1984) Lysis of Vibrio cholerae cells: direct isolation of the outer membrane from whole cells by treatment with urea. J Gen Microbiol 130:2027-2033

Lugtenberg B, Van Alphen L (1983) Molecular architecture and functioning of the outer membrane of Escherichia coli and other Gram-negative bacteria. Biochim Biophys Acta 737:51-115

Malinverni JC, Werner J, Kim S, Sklar JG, Kahne D, Misra R, Silhavy TJ (2006) YfiO stabilizes the YaeT complex and is essential for outer membrane protein assembly in Escherichia coli. Mol Microbiol 61:151-164

Moriel DG, Bertoldi I, Spagnuolo A, Marchi S, Rosini R, Nesta B, Pastorello I, Corea VA, Torricelli G, Cartocci E, Savino S, Scarselli M, Dobrindt U, Hacker J, Tettelin H, Tallon LJ, Sullivan S, Wieler LH, Ewers C, Pickard D, Dougan G, Fontana MR, Rappuoli R, Pizza M, Serino L (2010) Identification of protective and broadly conserved vaccine antigens from the genome of extraintestinal pathogenic Escherichia coli. Proc Natl Acad Sci USA 107:9072-9077

Morris DC, Peng F, Barker JR, Klose KE (2008) Lipidation of an FlrC-dependent protein is required for enhanced intestinal colonization by Vibrio cholera. J Bacteriol 190:231-239

Nakayama H, Kurokawa K, Lee BL (2012) Lipoproteins in bacteria: structures and biosynthetic pathways. FEBS J 279:4247-4268

Narita S, Tokuda H (2007) Amino acids at positions 3 and 4 determine the membrane specificity of Pseudomonas aeruginosa lipoproteins. J Biol Chem 282:13372-13378

Okuda S, Tokuda H (2011) Lipoprotein sorting in bacteria. Annu Rev Microbiol 65:239-259

Onufryk C, Crouch ML, Fang FC, Gross CA (2005) Characterization of six lipoproteins in the $\sigma^{\mathrm{E}}$ regulon. J Bacteriol 187:4552-4561

Paulsen IT, Brown MH, Skurray RA (1996) Proton-dependent multidrug efflux systems. Microbiol Rev 60:575-608

Schulze RJ, Zückert WR (2006) Borrelia burgdorferi lipoproteins are secreted to the outer surface by default. Mol Microbiol 59:1473-1484

Schulze RJ, Chen S, Kumru OS, Zückert WR (2010) Translocation of Borrelia burgdorferi surface lipoprotein OspA through the outer membrane requires an unfolded conformation and can initiate at the C-terminus. Mol Microbiol 76:1266-1278

Skare JT, Shang ES, Foley DM, Blanco DR, Champion CI, Mirzabekov T, Sokolov Y, Kagan BL, Miller JN, Lovett MA (1995) Virulent strain associated outer membrane proteins of Borrelia burgdorferi. J Clin Invest 96:2380-2392

Sutcliffe IC, Harrington DJ, Hutchings MI (2012) A phylum level analysis reveals lipoprotein biosynthesis to be a fundamental property of bacteria. Protein Cell 3:163-170

Tanaka K, Matsuyama SI, Tokuda H (2001) Deletion of lolB, encoding an outer membrane lipoprotein, is lethal for Escherichia coli and causes accumulation of lipoprotein localization intermediates in the periplasm. J Bacteriol 183:6538-6542

Terada M, Kuroda T, Matsuyama SI, Tokuda H (2001) Lipoprotein sorting signals evaluated as the LolA-dependent release of lipoproteins from the cytoplasmic membrane of Escherichia coli. J Biol Chem 276:47690-47694

Wai SN, Lindmark B, Söderblom T, Takade A, Westermark M, Oscarsson J, Jass J, Richter-Dahlfors A, Mizunoe Y, Uhlin BE (2003) Vesicle-mediated export and assembly of poreforming oligomers of the enterobacterial ClyA cytotoxin. Cell 115:25-35

Wensink J, Witholt B (1981) Outer-membrane vesicles released by normally growing Escherichia coli contain very little lipoprotein. Eur J Biochem 116:331-335 
Wilson MM, Bernstein HD (2016) Surface-exposed lipoproteins: an emerging secretion phenomenon in gram-negative bacteria. Trends Microbiol 24:198-208

Wright AC, Powell JL, Kaper JB, Morris JG Jr (2001) Identification of a group 1-like capsular polysaccharide operon for Vibrio vulnificus. Infect Immun 69:6893-6901

Wu T, McCandlish AC, Gronenberg LS, Chng SS, Silhavy TJ, Kahne D (2006) Identification of a protein complex that assembles lipopolysaccharide in the outer membrane of Escherichia coli. Proc Natl Acad Sci USA 103:11754-11759
Yakushi T, Masuda K, Narita S, Matsuyama S, Tokuda H (2000) A new ABC transporter mediating the detachment of lipidmodified proteins from membranes. Nat Cell Biol 2:212-218

Yamaguchi K, Yu F, Inouye M (1988) A single amino acid determinant of the membrane localization of lipoproteins in E. coli. Cell 53:423-432

Zhang YJ, Chen G, Lin H, Wang P, Kuang B, Liu J, Chen S (2017) Development of a regulatable expression system for the functional study of Vibrio vulnificus essential genes. Antonie Van Leeuwenhoek 110(4):607-614 